\title{
Multi Objective For Optimal Reactive Power Flow Using Modified PSO Considering TCSC
}

\author{
N. Mancer, B. Mahdad", K. Srairi, M. Hamed \\ Department of Electrical Engineering, University of Biskra, Biskra (07000), Algeria
}

\begin{abstract}
Multi objective optimal reactive power flow considering FACTS technology is becoming one of the most important issue in power system planning and control. This paper presents a new variant of particle swarm algorithm with time varying acceleration coefficients (TVAC) to solve multi objective optimal reactive power flow (MOORPF) (power loss minimization and voltage deviation). The proposed algorithm is used to adjust dynamically the parameters setting of Thyristor controlled series capacitor (TCSC) in coordination with voltages of generating units. This study is implemented on the standard IEEE 30-Bus system and the results are compared with other evolutionary programs such as simple genetic algorithm (SGA) and the simple particle swarm algorithm (SPSO). Simulation results confirm robustness of this new variant based PSO in term of solution quality and convergence time.
\end{abstract}

Keywords Optimal Reactive Power Flow (ORPF), Multi Objective, Loss Minimization, Voltage Deviation, TCSC, PSO, Time Varying Acceleration Coefficient (TVAC)

\section{Introduction}

Optimal reactive power flow (ORPF) plays an important role in optimal operation problems of power system[1], which referred to assign certain variable such generator voltage, tap ratios of transformers, shunt capacitor or reactor to minimize the real power loss, voltage deviation or any other objective function. This optimization should satisfy a given set of constraints which come from physical and operational limitations.

Many classic optimization techniques such linear and non-linear programming[2], quadratic programming[3], and interior point method[4] have been applied for solving ORPF problem. These methods are incapable in handling non-linear, discontinuous function and constraints, and problems having multiple local minimum points.

Recently, stochastic search methods have been used widely for the global optimization problem. These method have been successfully used to solve the ORPF problem. In[5] Lee used simple genetic algorithm (SGA) to solve reactive power operational problem, Mahdad in[6] proposed the application of GA for Optimal Power Flow with Consideration of FACTS devices. Particle swarm optimization (PSO) was applied by Yoshida in[7] for reactive power and voltage control. Krishna in[8] proposed a solution to the economic power dispatch problem with PSO

\footnotetext{
* Corresponding author:

bemahdad@mselab.org (B. Mahdad)

Published online at http://journal.sapub.org/ijee

Copyright (C) 2012Scientific \& Academic Publishing. All Rights Reserved
}

with time varying acceleration coefficients for non-convex objective functions. The classical particle swarm optimization (PSO) first introduced by Kennedy and Eberhart 1995[9], this method applied with success to solve the reactive power planning, PSO developed through simulation of a simplified social system, and has been found to be robust and flexible in solving optimization problem. because this technique can generate a high-quality solution within shorter calculation time and stable convergence characteristic than other stochastic methods.

The concept of Flexible AC Transmission Systems (FACTS) was first defined by N.G. Hingorani, in 1988[10]. A Flexible Alternating Current Transmission System (FACTS) is defined by the IEEE as "a power electronic based system and other static equipment that provide control of one or more $\mathrm{AC}$ transmission system parameters to enhance controllability and increase power transfer capability"[11]. FACTS devices can be utilized to increase the transmission capacity, improve the stability and dynamic behaviours or ensure better power quality in modern power systems in comparison to conventional devices like switched compensation. Hence, there is an interest in better utilization of available capacities bay installing flexible AC transmission systems (FACTS) devices[10-15] such as Thyristor controlled series compensators (TCSC). The TCSC is an important member of the FACTS family. It allows rapid and continuous changes of the transmission line impedance[12-14]. Active power flow across the compensated transmission line can be maintained at a specified level under a wide range of operating conditions. This paper investigates the application of PSO algorithm and 
the advantage of the TCSC for reactive power dispatch to minimize real power loss in transmission network and reduce the voltage deviation.

In this work a new variant of PSO based on time varying acceleration coefficient (TVAC) is proposed to solve the multi objective optimal reactive power planning considering TCSC Controllers. The proposed algorithm is validated on the standard IEEE 30-Bus test system and compared to SGA and SPSO. Simulation results confirm efficiency of this variant in term of solution quality and convergence time.

\section{Multi-Objective ORPF Formulation}

The multi objective ORPF is to optimize the settings of control variables in terms of one or more objective functions while satisfying several equality and inequality constraints. In multi objective ORPF we have to optimize two or more objective functions simultaneously. The problem can be formulated as:

$$
\begin{array}{ll}
\text { Minimize: } & J_{i}(x, u) \quad i=1, \ldots, N_{o b j} \\
\text { Subject to: } & g(x, u)=0 \\
& h(x, u)=0
\end{array}
$$

Where $J_{i}$ is the ith objective function, and $N_{o b j}$ is the number of objectives. $G$ is the equality constraints, $h$ is the system operation constraints. The main objective of this work is to optimize two competing objective functions, power loss and voltage deviation, while satisfying several equality and inequality constraints. The function can be written in the following[16]:

$$
F=\min \left((1-U) * f_{1}+U * f_{2}\right), U \in[0,1]
$$

Where: $f_{1}, f_{2}$ the two objective functions to be optimized simultaneously.

\subsection{Objectives Functions}

\subsubsection{Real Power loss (RPL)}

The first objective of the reactive power optimization is to minimize the real power loss in the transmission network, which is defined as follows:

$$
\begin{gathered}
f 1=\min (R P L)=\min \quad P_{\text {loss }}\left(x_{1}, x_{2}\right) \\
=\sum_{k=1}^{n l} g_{k}\left[V_{i}^{2}+V_{j}^{2}-2 * V_{i} V_{j} \cos \theta_{i j}\right]
\end{gathered}
$$

Where $P_{l o s s}$ is the active power loss, $x_{1}$ is the control variable vector $\left[V_{G}, X_{T C S C}\right]$ represented the generator voltage (continuous) and the series capacitor/inductor respectively, $\mathrm{x} 2$ is the dependent variable vector $\left[V_{L}, Q_{G}\right]$, $V_{L}$ is the load-bus voltage, $Q_{G}$ is the generator reactive power, $g_{k}$ is the conductance of branch between bus $i$ and $\mathrm{j}$, $V_{i}, V_{j}$ is the voltage at bus $\mathrm{i}-\mathrm{j}$.

\subsubsection{Voltage Deviation (VD)}

This objective function is to minimize the deviations in voltage magnitudes at load bus that can be expressed through the following equation:

$$
f_{2}=\min V D=\sum_{i=1}^{n p q}\left|V_{i}-1.0\right|
$$

\section{A. Constraints:}

The equality constraints represent the power flow equations

$$
\begin{aligned}
& P_{i}-V_{i} \sum_{j}^{N b} V_{j}\left(G_{i j} \cos \theta_{i j}+B_{i j} \sin \theta_{i j}\right)=0 \\
& Q_{i}-V_{i} \sum_{j=1}^{N b} V_{j}\left(G_{i j} \sin \theta_{i j}-B_{i j} \cos \theta_{i j}\right)=0 \\
& i=1: N b-1
\end{aligned}
$$

where $V_{j}$ is the voltage at bus $j, \theta_{i j}$ is the voltage angle difference between bus i and $j, G_{i j}$ is the conductance between bus $i$ and $j, B_{i j}$ is the susceptance between bus $i$ and $j, N_{b}$ is the set of numbers of buses, $N_{p q}$ is the set of numbers of total buses load bus.

The inequality constraints of the system consist of the upper and lower limits of active power generation of slack bus, load bus voltage, control variable limit, reactive power generation and the Shunt FACTS parameters which are described by:

$$
\begin{gathered}
P_{g, \text { slack }}^{\min } \leq P_{g, \text { slack }} \leq P_{g, \text { slack }}^{\max } \\
V_{L, 1}^{\min } \leq V_{L, 1} \leq V_{L, 1}^{\max } \\
V_{G, i}^{\min } \leq V_{G, i} \leq V_{G, i}^{\max } \\
Q_{G, i}^{\min } \leq Q_{G, i} \leq Q_{G, i}^{\max } \\
-0.2 * X_{l} \leq X_{T C S C} \leq 0.2 * X_{l}
\end{gathered}
$$

\section{Modeling of TCSC}

The TCSC is a series compensation component which consists of a series capacitor bank shunted by Thyristor controlled reactor as presented in Fig.1.

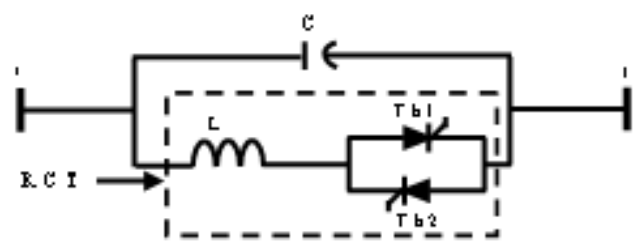

Figure 1. Basic TCSC configuration

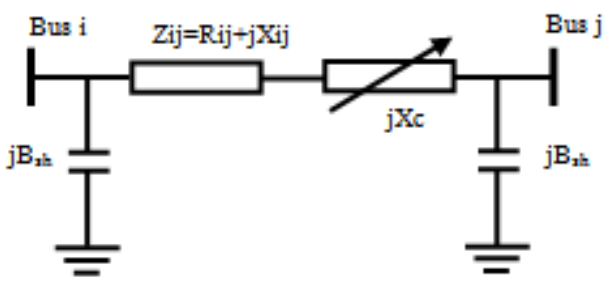

Figure 2. Single line diagram of TCSC model

The basic idea behind power flow control with the TCSC is to decrease or increase the overall lines effective series transmission impedance, by adding a capacitive or inductive reactive correspondingly. The TCSC is modelled as variable 
impedance[12-14]. The basic TCSC configuration is shown in Fig. 1. After installing TCSC, the new reactance of TCSC is presented by:

$$
X_{T C S C}=(1-k) X_{\text {line }}
$$

Where $\mathrm{X}$ is the transmission line reactance and $\mathrm{k}$ is the level of reactance compensation. The level of the applied compensation of the TCSC varies generally between $20 \%$ in inductive mode and $20 \%$ in capacitive mode[13]. Fig.2 shows the simplified single line diagram of TCSC model.

\section{PSO Strategy Based Time Varying Acceleration Coefficients}

Since the introduction of PSO method, a number of different PSO strategies are being applied by researchers for solving the ORPF, and other complex problem.

PSO is a based modern heuristic search method motivated from the simulation of the behaviour of social systems such as fish schooling and birds flocking[8-9]. The motivation behind this concept is to well balance the exploration and exploitation capability for attaining better convergence to the optimal solution. The PSO beginning, a population of particles is initialized with random positions marked by vectors $x i$ and random velocities vi, population of such particles is called a "swarm". The particles update their positions using their own experience and the experience of their neighbors. The update mode is termed as the velocity of particles[17].

The modified velocity and position of each particle can be calculated using the current velocity and the distance from Pbest $_{i}$ to Gbest $t_{i}$ as shown in the following formulas general:

$$
\begin{gathered}
\left\{\begin{array}{c}
V(t+1)=w^{*} V(t)+C_{1} * R_{1} *\left(\text { Pbest }_{i}-X(t)\right) \\
+C_{2} * R_{2} *\left(\text { Gest }_{i}-X(t)\right)
\end{array}\right. \\
X(t+1)=X(t)+V(t+1)
\end{gathered}
$$

where $\mathrm{V}(\mathrm{t})$ is the current velocity, $\mathrm{V}(\mathrm{t}+1)$ is the velocity (modified velocity) $\mathrm{R} 1$ and $\mathrm{R} 2$ are the random numbers between 0 and 1 , Pbesti is the best value found by particle $i$, Gbesti is the best particle found in the group, $\mathrm{X}(\mathrm{t})$ is the current position $\mathrm{X}(\mathrm{t}+1)$ is the current position (modified searching point), Here $\mathrm{w}$ is the inertia weight parameter, $\mathrm{C} 1 ; \mathrm{C} 2$ are cognitive and social coefficients, A large inertia weight helps in good global search while a smaller value facilitates local exploration. The concept of time varying inertial weight was introduced in[8-17] is suggested to decrease linearly from 0.9 to 0.4 during the run the inertial weights formulated as in (17)

$$
w=\left(w_{\max }-w_{\min }\right) * \frac{\left(\text { iter }_{\max }-\text { iter }\right)}{\text { iter }_{\max }}+w_{\text {min }}
$$

Where iter is the current iteration number while itermax is the maximum number of iterations. The flow chart of the proposed algorithm based TCSC is described in Figure 3.

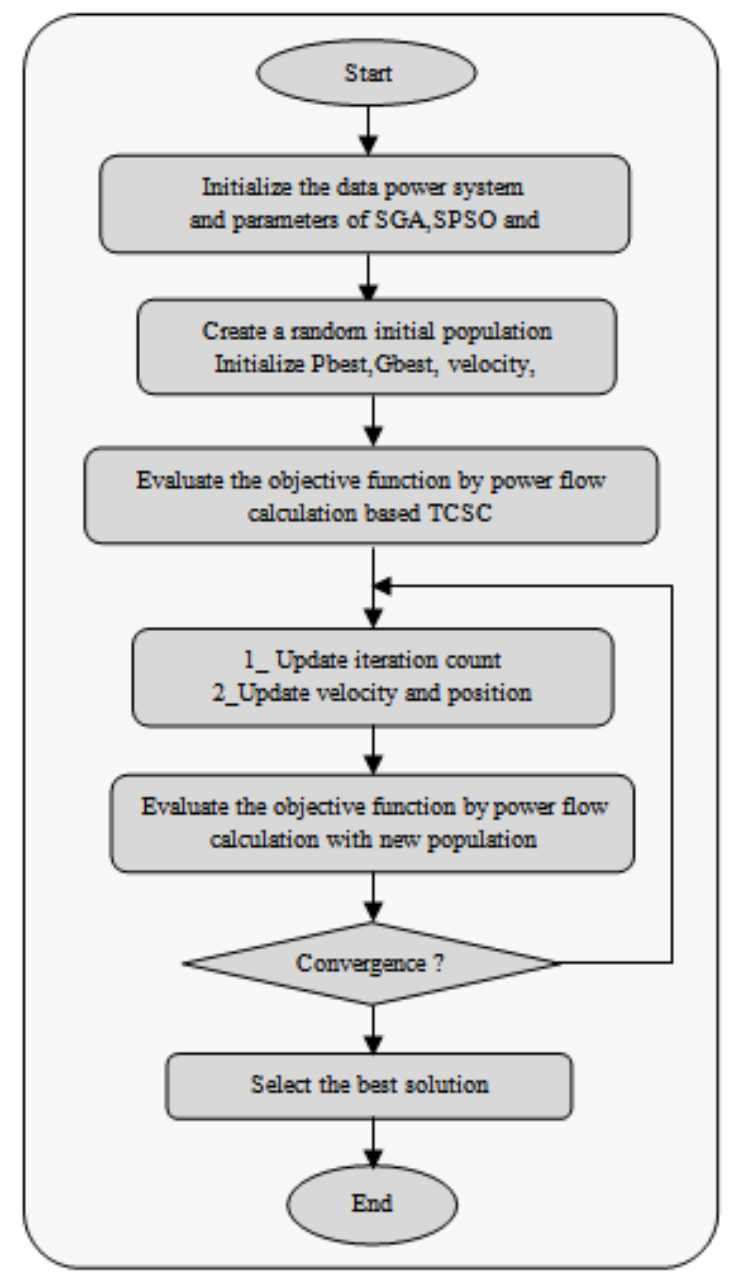

Figure 3. Flow Chart of the proposed algorithm based GA, SPSO and PSO-TVAC considering TCSC

Table 1. Parameters setting of the three algorithms

\begin{tabular}{|c|c|c|}
\hline SGA & SPSO & PSO-TVAC \\
\hline Population size 20 & Population size 20 & Population size 20 \\
\hline $\begin{array}{c}\text { Max- generations } \\
100\end{array}$ & $\begin{array}{c}\text { Max- generations } \\
100\end{array}$ & $\begin{array}{c}\text { Max- generations } \\
100\end{array}$ \\
\hline Selection $0.5 \%$ & $\mathrm{C} 1=1$ & $\mathrm{C} 1 \mathrm{f}=0.2, \mathrm{C} 1 \mathrm{i}=2.5$ \\
\hline Crossover rate $0.5 \%$ & $\mathrm{C} 2=3$ & $\mathrm{C} 2 \mathrm{f}=2.2, \mathrm{C} 2 \mathrm{i}=0.2$ \\
\hline Mutation 0.15 & - & $\begin{array}{l}\text { Wmin }=0.4, \\
\text { Wmax }=0.9\end{array}$ \\
\hline
\end{tabular}

\section{Simulation and Numerical Results}

The proposed algorithm is implemented and tested on a standard IEEE 30-Bus test system[18]; it consists of 6 generators located at buses $1,2,3,5,8,11$, and 13, 41 branches (lines) and four transformers in line 6-9, 6-10, 4-12, and 28-27. In this study in all cases one TCSC installed at the 
branch 27-28. The algorithms were implemented using the Matlab programming language; detailed analyses of the results are presented and discussed in this section.

\section{A. Testing Strategies}

Case 1: Single objective function: Ploss minimization with and without TCSC Controller.

The main goal of this first case is to verify the feasibility and performance of the new proposed PSO variant based time varying acceleration to solve the ORPF problem. Table 1 shows the parameters of SGA, SPSO and PSO-TVAC. Figs 4-5-6 show the convergence characteristics of active power loss for SGA, SPSO and PSO-TVAC.

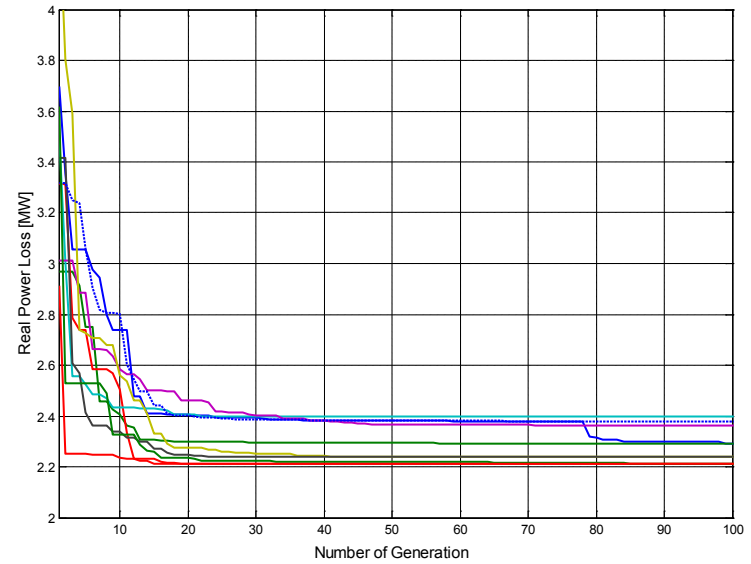

Figure 4. Convergence characteristic of real power loss based SGA

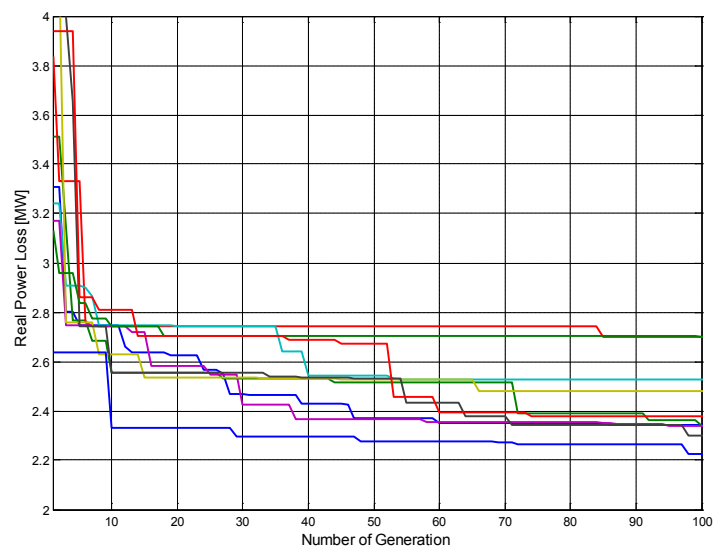

Figure 5. Convergence characteristic of real power loss based SPSO

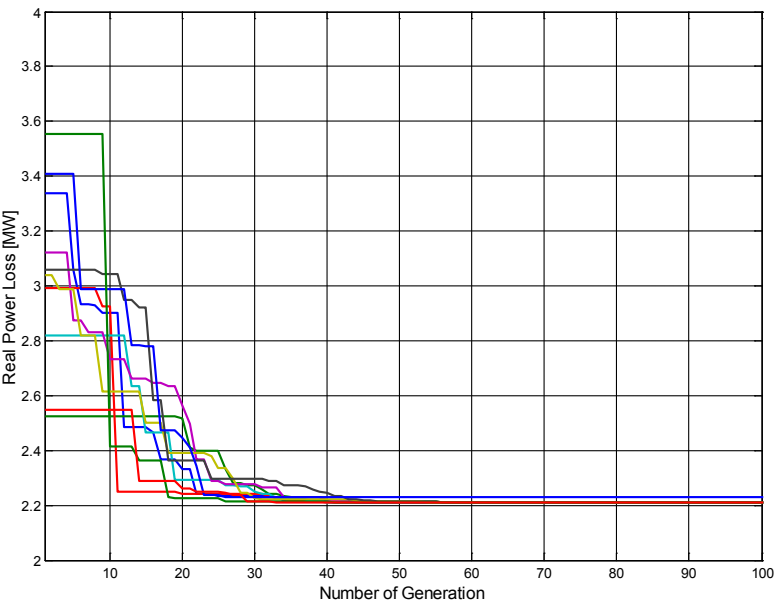

Figure. 6. Convergence characteristic of real power loss based PSO-TVAC

Table.2 illustrates the results of best real power transmission losses using PSO-TVAC compared to SGA and PSO with and without TCSC. It can be seen that the power loss optimized without considering TCSC controller using PSO_tvac is 2.2091 MW which is better than the result fond using SGA and SPSO. Figs 4-5-6 show the convergence characteristic of the SGA, SPSO and to the proposed PSO_ TVAC.

Case 2: Single objective function: VD minimization with and without TCSC Controller.

In this second case the objective function considered is related to voltage deviation, one TCSC installed at the same bus, the reactance of this series controller adjusted dynamically in coordination with voltage of generators to minimize the voltage deviation. Table. 3 illustrates the results of best real voltage deviation using PSO_TVAC compared to GA and PSO with and without TCSC. It can be seen that the voltage deviation optimized without considering TCSC controller using PSO_TVAC is 0.1356 p.u which is better than the result fond using others basic optimization methods. Fig 7 shows Convergence characteristic of voltage deviation used PSO_TVAC Compared to SGA, SPSO. Based on Figs 4-5-6, it is clear that the

Table 2. Results of best real power transmission losses using PSO_TVAC compared to GA, PSO, with and without TCSC

\begin{tabular}{|c|c|c|c|c|c|c|}
\hline & \multicolumn{3}{|c|}{ Min P loss Without TCSC } & \multicolumn{3}{c|}{ Min P loss With TCSC } \\
\cline { 2 - 7 } & SGA & SPSO & PSO TVAC & SGA & SPSO & PSO TVAC \\
\hline V1 (p.u) & 1.0000 & 1.0000 & 1.0000 & 1.0000 & 1.0000 & 1.0000 \\
\hline V2 (p.u) & 1.0012 & 1.0008 & 1.0011 & 1.0012 & 1.0078 & 1.0011 \\
\hline V5 (p.u) & 1.0757 & 1.0850 & 1.0746 & 1.0749 & 1.1000 & 1.0718 \\
\hline V8 (p.u) & 1.0176 & 1.0307 & 1.0186 & 1.0192 & 1.0295 & 1.0168 \\
\hline V11 (p.u) & 1.0310 & 1.0396 & 1.0329 & 1.0333 & 1.0503 & 1.0309 \\
\hline V13 (p.u) & 1.0365 & 1.0416 & 1.0377 & 1.0357 & 1.0359 & 1.0340 \\
\hline X_TCSC (p.u) & - & - & - & 0.0800 & 0.0800 & 0.0800 \\
\hline DP max (m.w) & 2.3987 & 2.7006 & 2.2280 & 2.2857 & 2.6559 & 2.2398 \\
\hline DP_average(m.w) & 2.2835 & 2.4333 & 2.2110 & 2.2154 & 2.4172 & 2.2070 \\
\hline DP min(m.w) & 2.2100 & 2.2266 & 2.2091 & 2.1944 & 2.2538 & 2.1939 \\
\hline DV (p.u) & 0.2706 & 0.3713 & 0.2774 & 0.2658 & 0.3834 & 0.2518 \\
\hline Time (s) & 0.0249 & 0.0214 & 0.0202 & 0.0252 & 0.0224 & 0.0210 \\
\hline
\end{tabular}


Table 3. The results of best voltage deviation using PSO_TVAC Compared to GA, PSO with and without TCSC

\begin{tabular}{|c|c|c|c|c|c|c|}
\hline & \multicolumn{3}{|c|}{ Min DV Without TCSC } & \multicolumn{3}{c|}{ Min DV With TCSC } \\
\cline { 2 - 7 } & SGA & SPSO & PSO TVAC & SGA & SPSO & PSO TVAC \\
\hline V1 (p.u) & 1.0000 & 1.0000 & 1.0000 & 1.0000 & 1.0000 & 1.0000 \\
\hline V2 (p.u) & 1.0302 & 1.0358 & 1.0351 & 1.0380 & 1.0297 & 1.0357 \\
\hline V5 (p.u) & 1.0475 & 1.0154 & 1.0409 & 1.0357 & 0.9823 & 1.0414 \\
\hline V8 (p.u) & 1.0129 & 1.0115 & 1.0118 & 1.0114 & 1.0310 & 1.0116 \\
\hline V11 (p.u) & 0.9996 & 1.0418 & 1.0063 & 1.0114 & 1.0666 & 1.0059 \\
\hline V13 (p.u) & 1.0310 & 1.0278 & 1.0200 & 1.0200 & 1.0198 & 1.0212 \\
\hline X_TCSC (p.u) & - & - & - & 0.0800 & -0.018 & 0.0800 \\
\hline DV max (p.u) & 0.1807 & 0.2605 & 0.1639 & 0.2845 & 0.2587 & 0.1375 \\
\hline DV_average (p.u) & 0.1510 & 0.1776 & 0.1401 & 0.1661 & 0.2052 & 0.1364 \\
\hline DV min(p.u) & 0.1399 & 0.1468 & 0.1360 & 0.1376 & 0.1751 & 0.1356 \\
\hline DP (m.w) & 3.0493 & 3.3875 & 3.2090 & 3.3484 & 3.9210 & 3.2538 \\
\hline Time (s) & 0.0093 & 0.0221 & 0.0199 & 0.0247 & 0.0212 & 0.0223 \\
\hline
\end{tabular}

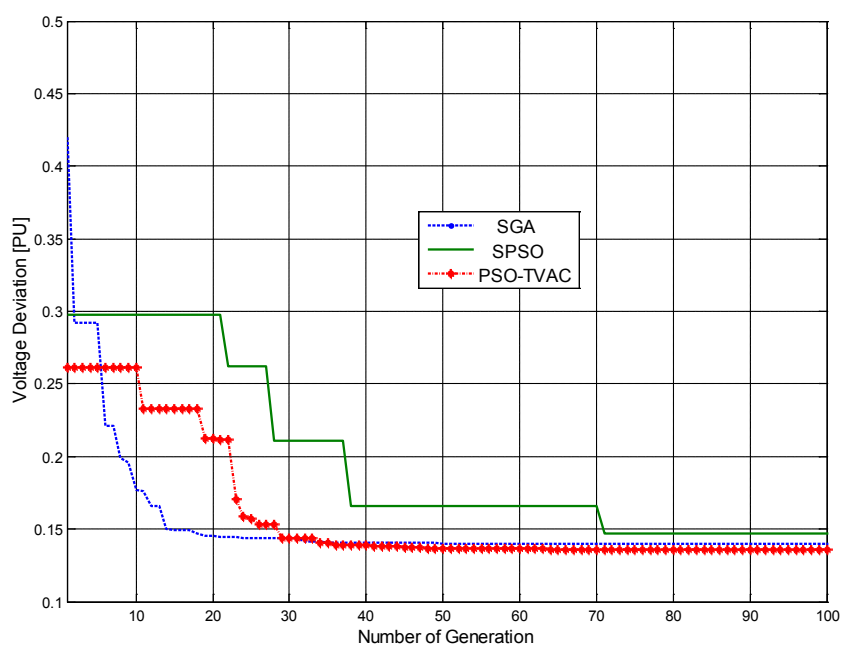

Figure 7. Convergence characteristics for voltage deviation using PSO-TVAC, GA and PSO

Case 3: Multi Objective optimization: Ploss and VD minimization considering TCSC Controller.

In order to verify the efficiency of the proposed PSO_TVAC variant, voltage control of generating and reactance of TCSC installed at specified bus considered to optimize simultaneously two objective functions (voltage deviation and transmission power losses). Fig 8 shows the Pareto optimal solution results using the proposed PSO_TVAC variant. The simulation results show clearly that PSO_TVAC leads to lower active power loss (2.2096 MW) and voltage deviation ( 0.2414 p.u) compared to the others standard optimization methods.

Table 5. The results of the combined best power loss and voltage deviation using PSO_TVAC compared to GA, PSO considering One TCSC

\begin{tabular}{|c|c|c|c|}
\hline & \multicolumn{3}{|c|}{ Min Ploss \& Min DV With One TCSC } \\
\hline & SGA & SPSO & PSO_TVAC \\
\hline V1 (p.u) & 1.0000 & 1.0000 & 1.0000 \\
\hline V2 (p.u) & 0.9996 & 0.9980 & 1.0027 \\
\hline V5 (p.u) & 1.0624 & 1.0581 & 1.0676 \\
\hline V8 (p.u) & 1.0122 & 1.0129 & 1.0152 \\
\hline V11 (p.u) & 1.0247 & 1.0245 & 1.0280 \\
\hline V13 (p.u) & 1.0318 & 1.0327 & 1.0310 \\
\hline X_TCSC (p.u) & 0.0800 & 0.0668 & 0.0800 \\
\hline DRPL (m.w) & 2.2088 & 2.2167 & 2.2096 \\
\hline DV (p.u) & 0.2563 & 0.2638 & 0.2414 \\
\hline
\end{tabular}

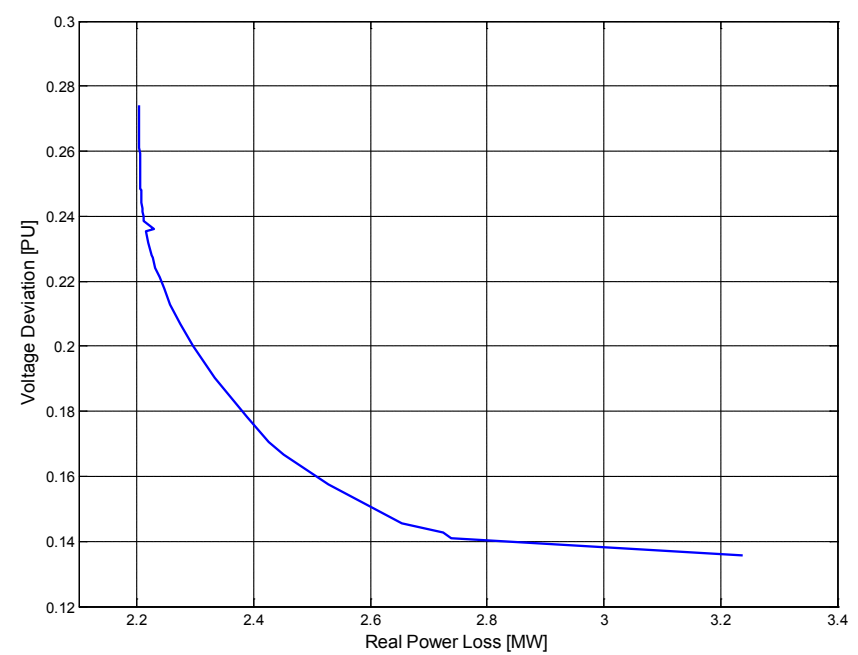

Figure 8. Pareto optimal solution results based PSO-TVAC

\section{Conclusions}

This paper presents the application of modified variant of PSO using time varying acceleration to adjust dynamically the reactance of TCSC controller in coordination with voltage control of generators to improve the multi-objective ORPF solution. Comparing the proposed algorithm with two other techniques (SGA and PSO) shows the advantage of this algorithm in decreasing transmission loss and voltage deviation. Also the results obtained demonstrate the performances of the proposed approach based PSO-TVAC in term of solution quality and convergence characteristic.

\section{REFERENCES}

[1] Zhang X, Chen W, Dai C, Cai W. "Dynamic multi-group self-adaptive differential evolution algorithm for reactive power optimization', Elect Power and energy syst, 2010, 32,pp.351-357.

[2] N. Deeb, S.M. Shahidepour, Linear reactive power optimization in a large power network using the decomposition approach, IEEE Trans. Power Syst. 5 (2) (1990) 428-435. 
[3] J.A. Momoh, S.X. Guo, E.C. Ogbuobiri, R. Adapa, The quadratic interior point method solving power system optimization problems, IEEE Trans.Power Syst. 9 (3) (1994) $1327-1336$.

[4] S. Granville, Optimal reactive dispatch through interior point methods, IEEE Trans. Power Syst. 9 (1) (1994) 136-146.

[5] Lee KY,Bai X,Park YM. ' optimization methode for reactive power planning by using a modified simple genetic algorithm'. IEEE trans power syst, vol 10 no, 4; 1995 p1843-50.

[6] B. Mahdad, Optimal Power Flow with Consideration of FACTS devices Using Genetic Algorithm: Application to the Algerian Network, Doctorat Thesis, Biskra University Algeria, 2010.

[7] Yoshida H, Fukuyama Y, Kawata K, Takayama S, Nakanishi Y. "A particle swarm optimization for reactive power and voltage control considering voltage security assessment," IEEE Trans Power Syst 2001,15(4),pp1232-1239.

[8] TC Krishna, P Manjaree, S Laxmi.' Particle swarm optimization with time varying acceleration coefficients for non-convex economic power dispatch', , Elect Power and energy syst, 2009, 31,pp.249-257.

[9] J Kennedy, R Eberhart., "Particle swarm optimization" In: Proceedings of the IEEE conference on neural networks (ICNN'95), vol. IV. Perth, Australia; 1995. pp.1942-1948.

[10] N.G. Hingorani, L. Gyugyi, ' Understanding FACTS Concepts and Technology of Flexible AC Transmission
Systems", IEEE Press, New York, 2000.

[11] K. R. Padiyar . 'FACTS controllers in power transmission and distribution "', New age international publishers, 2007.

[12] R.Benabid, M.Boudour,M.A Abido.' Optimal location and setting of SVC and TCSC devices using non-dominated sorting particle swaem optimization". Elect Power and energy syst, 2009, 79,pp.1668-1677.

[13] K. shanmukha Sundar, H.M. Ravikumar,'sselection of TCSC location for securd optimal power flow under normal and network contingencies"' Elect Power and Energ Syst, 2012, 34,pp 29-37.

[14] P. L. So, Y. C. Chu, T. Yu, "Coordinated control of TCSC and SVC for system damping enhancement", International Journal of Control, Automation, and Systems, June $2005 \mathrm{Vol}$. 3, No. 2, pp 322-33.

[15] B. Mahdad "Contribution to the improvement of power quality using multi hybrid model based Wind-Shunt FACTS,' 10th EEEIC International Conference on Environment and Electrical Engineering, Italy, 2011.

[16] M.A. Abido. "A novel multiobjective evolutionary algorithm for environmental/economic power dispatch" Elect Power Syst Res, 2003, 65,pp 71-81.

[17] RC. Eberhart, Y. Shi. 'Comparing inertia weights and constriction factors in particle swarm optimization'. Proc Congr Evol Comput 2000;1,pp 84-88

[18] www.pserc.cornell.edu/matpower/, Package of MATLAB, MATPOWER 